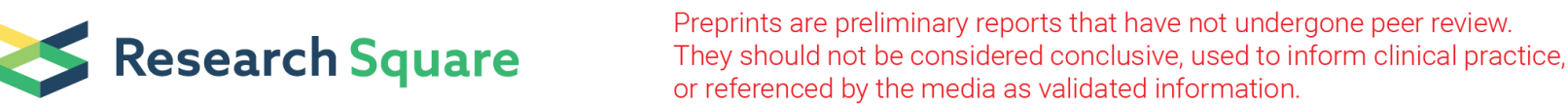

\section{An Integrated Analysis Reveals Geniposide Extracted From Gardenia Jasminoides Ellis Regulates Calcium Signaling Pathway Essential for Influenza a Virus Replication}

\section{LiRun Zhou}

China Academy of Chinese Medical Sciences https://orcid.org/0000-0002-1350-940X

\section{Lei Bao}

China Academy of Chinese Medical Sciences

\section{Yaxin Wang}

China Academy of Chinese Medical Sciences

\section{Mengping Chen}

China Academy of Chinese Medical Sciences

\section{Yingying Zhang}

Beijing University of Chinese Medicine Affiliated Dongzhimen Hospital

\section{Zihan Geng}

China Academy of Chinese Medical Sciences

\section{Ronghua Zhao}

China Academy of Chinese Medical Sciences

\section{Jing Sun}

China Academy of Chinese Medical Sciences

\section{Yanyan Bao}

China Academy of Chinese Medical Sciences

\section{Yujing Shi}

China Academy of Chinese Medical Sciences

\section{Rongmei Yao}

China Academy of Chinese Medical Sciences

Shanshan Guo ( $\nabla$ ssguo@icmm.ac.cn )

China Academy of Chinese Medical Sciences

Xiaolan Cui

China Academy of Chinese Medical Sciences

Research 
Keywords: Geniposide, Influenza A virus, RNA polymerase, Calcium signaling pathway, CAMKII

Posted Date: August 9th, 2021

DOI: https://doi.org/10.21203/rs.3.rs-764303/v1

License: (c) (1) This work is licensed under a Creative Commons Attribution 4.0 International License. Read Full License 


\section{Abstract}

\section{Background}

Geniposide, an iridoid glycoside purified from the fruit of Gardenia jasminoides Ellis, has been reported to possess pleiotropic activity against different diseases. In particular, geniposide possesses a variety of biological activities and exerts good therapeutic effects in the treatment of several strains of the influenza virus. However, the molecular mechanism for the therapeutic effect has not been well defined.

\section{Methods}

This study aimed to investigate the mechanism of geniposide on influenza A virus (IAV). The potential targets and signaling pathways of geniposide in the IAV infection were predicted using network pharmacology analysis. According to the result of network pharmacology analysis, we validated the calcium signaling pathway induced by IAV and investigated the effect of geniposide extracted from Gardenia jasminoides Ellis on this pathway.

Results

The primary gene ontology (GO) biological processes and Kyoto Encyclopedia of Genes and Genomes (KEGG) pathways KEGG enrichment analysis indicated that geniposide has a multi-target and multipathway inhibitory effect against influenza, and one of the mechanisms involves calcium signaling pathway. In the current study, geniposide treatment greatly decreased the levels of RNA polymerase in HEK-293T cells infected with IAV. knocking down CAMKII in IAV-infected HEK-293T cells enhanced virusRNA (vRNA) production. Geniposide treatment increased CAMKII expression after IAV infection. Meanwhile, the CREB and c-Fos expressions were inhibited by geniposide after IAV infection. The experimental validation data showed that the geniposide were able to alleviated extracellular Ca2+ influx, dramatically decreased neuraminidase activity and suppressed IAV replication in vitro via regulating the calcium signaling pathway.

Conclusions

These anti-IAV effects might be related to the disrupted interplay between IAV RNA polymerase and CAMKII and the regulation of the downstream calcium signaling pathway essential for IAV replication. Taken together, the findings reveal a new facet of the mechanism by which geniposide fights IAV in a way that depends on CAMKII replication.

\section{Background}

Influenza A viruses (IAV) is common in the all age group population. It has been regarded as a severe public health. It is one of the most common infectious diseases with a high incidence rate and high mortality rate [1]. Although there are 26 licensed inactivated vaccines that are available for the prevention of influenza, but the number of the death caused by epidemics worldwide is still estimated as 300,000- 
650,000 every year [2, 3]. As influenza A virus (IAV) rapidly evolves through antigenic drift and shift, new subtype strains continuously emerge [4,5]. For example, the most recent influenza pandemic caused by the H1N1 pdm09 influenza virus originated from swine, spread rapidly to nearly all countries and territories [6, 7]. Compared to seasonal influenza viruses, the H1N1 pdm09 influenza virus causes more severe disease and deaths among adults aged 18-64 years [8, 9]. Human infection with H7N9 influenza viruses of avian origin emerged in March 2013 in China, and these viruses have continued to spread into populations with unprecedented mortality and morbidity $[10,11]$. Obviously, Influenza has the potential for mortality, high mutation rates, and pandemic risk; thus, it is crucial to learn more about the virulence and pathogenicity of influenza and identify the targets for the development of new drugs [12].

Antiviral drugs play a critical role in defending against influenza epidemics and pandemics, especially against antigenically different strains or new subtypes [13]. Currently, the anti-influenza drugs approved by the United States Federal Drug Administration can be divided into two classes: adamantane-based M2 ion channel blockers, which inhibit viral replication by preventing endosome acidification and viral ribonucleoprotein delivery into the cytoplasm; and neuraminidase inhibitors, which inhibit the release of newly formed virus particles from infected cells $[14,15]$. However, surveillance research has indicated that almost all circulating human IAVs are resistant to adamantine, and the viruses rapidly develop resistance to neuraminidase inhibitors after their widespread application $[16,17]$. Given the inherent limitations of drugs targeting viral proteins, the development of novel drugs targeting cellular components may be a promising strategy [18].

The life cycle of the influenza virus involves host cellular proteins and pathways [19, 20]. The outcomes of influenza pathogenesis depend on the interactions between the virus and the host cellular proteins along with the activation of signal transduction pathways [21, 22]. The host cellular proteins and signaling pathways may facilitate virus replication by hijacking the host molecular machinery required for the viral life cycle or trigger the innate immune defense of the host to inhibit the virus [22]. $\mathrm{Ca}^{2+}$ influx may play a crucial role in the regulation of IAV entry and infection. Influenza A infection has been demonstrated to induce $\mathrm{Ca}^{2+}$ oscillation in host cells, and infection with influenza $\mathrm{A}$ is obviously attenuated by $\mathrm{Ca}^{2+}$ chelation [23]. In the early stage of infection, viral glycoprotein hemagglutinin binds to voltage-dependent $\mathrm{Ca}^{2+}$ channels on the host cell surface to induce intracellular $\mathrm{Ca}^{2+}$ oscillations, mediate the entry of IAV, and subsequently evoke the calcium signaling pathway of the host cell to facilitate infection [24].

Gardenia jasminoides Ellis (Rubiaceae), which is called Zhi-Zi in the Chinese pharmacopoeia, is an important heat-clearing and detoxifying Chinese herb [25] that has been used in the treatment of inflammation, acute febrile disease, ischemia/reperfusion injury, and hepatic disorders for a long time in East Asia [26-29]. Geniposide, a type of iridoid glycoside, is the main bioactive component isolated from Gardenia jasminoides Ellis [30]. Geniposide has diverse pharmacological activities, including antioxidant, anti-inflammatory, neuroprotective, and antithrombotic activities [31-34]. Moreover, accumulating evidence has demonstrated the antiviral properties of geniposide, which has been shown to inhibit infection by influenza A (H1N1) pdm09 virus, enterovirus 71 virus, and Epstein-Barr virus both in vitro 
and in vivo [35-37]. Our previous study showed that the iridoid glycoside extracted from Gardeniae fructus inhibited extracellular $\mathrm{Ca}^{2+}$ influx induced by IAV; however, the precise mechanism of action remains unclear [38].

In the current study, to better understand the antiviral mechanism of geniposide against influenza, we first constructed a protein-protein interaction (PPI) network of influenza virus-related molecules and then mapped geniposide-related genes to the network to reveal the correlation between them. After module identification, the primary gene ontology (GO) biological processes and Kyoto Encyclopedia of Genes and Genomes (KEGG) pathways were identified by enrichment analysis. Based on the results, we confirmed that the calcium signaling pathway is induced by the IAV and investigated the effect of geniposide extracted from Gardenia jasminoides Ellis on this pathway. Finally, the neuraminidase activity of IAV and extracellular $\mathrm{Ca}^{2+}$ influx was detected in Madin-Darby canine kidney (MDCK) cells, the expressions of proteins involved in calcium signaling [calcium/calmodulin-dependent protein kinase II (CAMKII), cAMP response element-binding protein (CREB), and c-Fos] were evaluated in A549 cells, and the RNA polymerase activity of influenza virus was determined in HEK-293T cells transfected with CAMK II siRNA.

\section{Material And Methods}

\subsection{Biosafety statement}

All experiments involving live IAV were carried out in the Animal Biosafety Level 2 Laboratory of the Institute of Chinese Materia Medica, China Academy of Chinese Medical Sciences.

\subsection{Cells and virus stock}

MDCK, A549, and HEK-293T cells were provided by the Cell Center of the Institute of Basic Medical Sciences, Peking Union Medical College (Beijing, China) and cultured in Dulbecco's modified Eagle's medium (Gibco) containing 10\% heat-inactivated fetal bovine serum (Gibco). For validation studies, the $A$ FM/1/47 (H1N1) influenza virus (ATCC-VR-1754-ATC) was used. The virus was propagated in 10-day-old embryonated chicken eggs (Merial Vital Laboratory Animal Technology Co., Ltd., Beijing, China) according to the method previously described [39]. The virus was titrated in MDCK cells and titers (median tissue culture infective dose, TCID50) determined to be 10-4.5 using the Reed-Muench method [40].

\subsection{Geniposide extracted from Gardenia jasminoides Ellis}

Geniposide with a purity of $39.4 \%$ was extracted from Gardenia jasminoides Ellis with $70 \%$ ethanol. The purity of iridoid glycoside exceeded $90 \%$, as detected by ultraviolet spectrophotometry.

\subsection{Acquisition of genes related to influenza virus and geniposide}

Genes related to influenza virus were searched in the OMIM database (https://www.ncbi.nlm.nih.gov/omim/). Geniposide was entered into the STITCH (http://stitch.embl.de/), 
GeneCards (https://www.genecards.org/), and CTD (http://ctdbase.org/) databases to search for related genes.

\subsection{Network construction and module identification}

Influenza virus-related molecules were determined based on the STRING database (Version 9.05). A network of the interactions among virus-related molecules and first-order neighbor proteins was constructed with Homo sapiens as the background. Geniposide-related genes were mapped to this network to understand the correlation between them. We used MCODE (http://baderlab.org/Software/MCODE), a clustering algorithm-based software, to identify gene network modules and the modularity of each network using the following parameters: degree cutoff $=2$, node score cutoff $=0.2$, max depth $=100$, and K-core threshold $=2$.

\subsection{Functional enrichment analysis}

In this study, DAVID 6.7 software (http://david.abcc.ncifcrf.gov/) was used for the functional enrichment analysis of each module, with species restricted to Homo sapiens. As primary GO biological process and KEGG pathway can describe the biological features of modules. Modified Fisher's exact test and the Benjamini-Hochberg method were utilized to calculate and correct the P-value $(P<0.05)$.

\subsection{Neuraminidase activity of IAV}

The antiviral activity of geniposide against IAV was evaluated by neuraminidase activity assay. MDCK cells were seeded in 96-well plates at a density of 1'105 cells/ml and infected with the FM/1/47 strain of the H1N1 influenza virus (100TCID50, $100 \mathrm{~mL} /$ well) for $1 \mathrm{~h}$. The infected cells were then treated with serially diluted geniposide solutions at concentrations of $320,160,80$, and $40 \mathrm{mg} / \mathrm{ml}$. Ribavirin served as a reference drug at a final concentration of $3.13 \mathrm{mg} / \mathrm{ml}$. After incubation at $37^{\circ} \mathrm{C}$ for $48 \mathrm{~h}$, the cells were harvested, and neuraminidase activity was determined using a neuraminidase assay kit (Beyotime, Shanghai, China) according to the manufacturer's protocol.

\subsection{Confocal microscopy}

MDCK cells were cultured on 35-mm glass-bottomed culture dishes (NEST, China) at a concentration of 5 ' 105 cells/ml. When the cells grew to $80 \%-90 \%$ confluence, a solution of influenza virus A/FM1/47 (100TCID50) was added to the cultures. After incubation at $37^{\circ} \mathrm{C}$ for $30 \mathrm{~min}$, the MDCK cells were loaded with $10 \mathrm{mg} / \mathrm{ml}$ of fluo-3 AM probe (Invitrogen) for $30 \mathrm{~min}$ to detect the intracellular calcium concentration. The cells were then imaged using a laser confocal microscope (Olympus, FV1000, Japan). To measure $\mathrm{Ca} 2+$ influx, the samples were excited by an argon laser at $488 \mathrm{~nm}$, and the fluorescence intensity of emission was detected at $530 \mathrm{~nm}$.

\subsection{Western blotting assay}


A549 cells at 12, 24, 36, and $48 \mathrm{~h}$ after infection with IAV/FM1/47 were lysed in RIPA lysate buffer supplemented with phenylmethylsulfonyl fluoride and protease inhibitor cocktail (Sigma). Total protein concentrations were determined using a bicinchoninic acid kit to ensure equal sample loading. Proteins were separated on $10 \%$ sodium dodecyl sulphate-polyacrylamide gel electrophoresis gel and then transferred onto a $0.45-\mu \mathrm{m}$ nitrocellulose membrane. After blocking with non-fat milk, the blots were incubated with the primary antibodies overnight at $4^{\circ} \mathrm{C}$ : CAMKII and glyceraldehyde-3-phosphate dehydrogenase (GAPDH; 1:2000, Abcam, UK, CREB, and c-Fos (1:1500, CST, USA). Subsequently, secondary antibody incubation was conducted with goat anti-rabbit IgG antibody (1:20000) for $3 \mathrm{~h}$ at room temperature. The blots were visualized by enhanced chemiluminescence, and the density of bands was determined using Image $\mathrm{J}$ software (National Institutes of Health, USA).

\subsection{Dual-luciferase reporter gene assay}

HEK-293T cells were transfected with CAMKII siRNA (forward primer, 5'- CACCACCAUUGAGGAGGAATT -3' and reverse primer, $5^{\prime}$-UUCCUCCUCAAUGGUGGUGTT- $3^{\prime}$ ) at $37^{\circ} \mathrm{C}$ under $5 \% \mathrm{CO} 2$. After $12 \mathrm{~h}$, the cells were co-transfected with four plasmids containing the cDNA of the RNA-dependent RNA polymerase (RdRP) of influenza virus A/WSN/33H1N1) (pHW181-PB2, pHW182-PB1, pHW183-PA, pHW185-NP) or the negative control pFlu-luc) using a lipofectamine 3000 transfection kit (Invitrogen, USA) according to a previous report [41]. After $12 \mathrm{~h}$ of transfection, the supernatant was discarded, and the cells were treated with different concentrations of geniposide ( 320 or $160 \mu \mathrm{g} / \mathrm{ml}$ ) for $24 \mathrm{~h}$ at $37^{\circ} \mathrm{C}$ and $5 \% \mathrm{CO} 2$. The luciferase activity was finally detected using a dual-luciferase reporter assay system (Promega, USA) according to the manufacturer's instructions. The relative luciferase activity was determined by the ratio of the Renilla luciferase value to the firefly luciferase value.

\subsection{Statistical analysis}

Experimental results are presented as the mean \pm standard error of the mean (SEM). Differences were analyzed by one-way analysis of variance using GraphPad Prism 7.0 software. Significant differences were determined by the Bonferroni test. Differences were considered to be significant at $P<0.05$.

\section{Results}

\subsection{Genes related to influenza virus and geniposide}

Based on the OMIM database, a total of 77 genes related to influenza viruses were obtained. A total of 35 genes related to geniposide were obtained from searches of the STITCH, GeneCards, and CTD databases.

\subsection{Network and module identification}

The PPI network constructed from virus-related molecules and first-order neighbors consisted of 2395 nodes and 5574 edges (score $\geq 0.9$ ). Geniposide-related genes were mapped to the PPI network, and 17 
were found to be associated with influenza virus (Fig. 1A). After MCODE analysis, we obtained nine modules consisting of more than three nodes from the PPI network, and the modules were sorted according to the scores (Fig. 1B, Table 1).

\subsection{Functional enrichment analysis of modules}

Based on DAVID 6.7 software (http://david.abcc.ncifcrf.gov/), Module 1 contained 14 pathways $(\mathrm{P}<$ 0.05): chemokine signaling pathway, neuroactive ligand-receptor interaction, taste transduction, cytokine-cytokine receptor interaction, gap junction, melanogenesis, progesterone-mediated oocyte maturation, intestinal immune network for IgA production, dilated cardiomyopathy, GnRH signaling pathway, complement and coagulation cascades, oocyte meiosis, vascular smooth muscle contraction, and calcium signaling pathway (Table 2$)$. Module 2 contained 11 pathways $(P<0.05)$ : toll-like receptor signaling pathway, RIG--like receptor signaling pathway, cytosolic DNA-sensing pathway, small-cell lung cancer, pathogenic Escherichia coli infection, NOD-like receptor signaling pathway, adipocytokine signaling pathway, pathways in cancer, epithelial cell signaling in Helicobacter pylori infection, pancreatic cancer, chronic myeloid leukemia (Table 3). Module 3 contained one pathway $(P<0.05)$ : proteasome (Table 4).

Module 1 is enriched in $326 \mathrm{GO}$ biological processes with 21 biological functional annotations: 52 for cell communication; 48 for metabolism; 40 for transport; 34 for signal transduction; 29 for nucleobase, nucleoside, nucleotide, and nucleic acid metabolism; 19 for biosynthesis; 19 for behavior; 19 for ion transport; 18 for response to external stimulus; 15 for response to stress; 12 for cell-cell signaling; 11 for response to endogenous stimulus; and 10 for protein modification $(P<0.05)$. Module 2 is enriched with $113 \mathrm{GO}$ biological processes $(\mathrm{P}<0.05)$, while Module 3 is enriched with $64 \mathrm{GO}$ biological processes $(\mathrm{P}<$ 0.05; Fig. 2).

To verify the results of the $G O$ analysis, the genes of the calcium signaling pathway in Module 2 were enriched by KEGG pathway analysis (Fig. 3).

\subsection{Geniposide inhibits the neuraminidase activity of IAV}

Neuraminidase plays a pivotal role during the final stages of influenza virus infection. Neuraminidase facilitates the release of progeny virus and the spread of virus from infected cells to neighboring ones. To explore the inhibitory effect of geniposide on IAV, the neuraminidase activity in MDCK cells was analyzed at $48 \mathrm{~h}$ after infection. In the virus control group, the neuraminidase activity increased dramatically compared to the cell control group. Geniposide treatment at concentrations of 320,160 and $80 \mu \mathrm{g} / \mathrm{ml}$ dramatically decreased the neuraminidase activity (Fig. 4A). These results demonstrate that geniposide significantly suppressed IAV in MDCK cells. 


\subsection{Geniposide inhibits IAV polymerase activity in HEK-293T cells}

Above, we demonstrated the inhibitory effect of geniposide on IAV. CAMKII is a crucial protein in the calcium signaling network. To clarify whether geniposide inhibits virus replication in a calcium-dependent manner, the IAV polymerase activity was determined in HEK-293T cells in which CAMKII protein expression was depleted by siRNA. When CAMKII was knocked down, the RNA polymerase activity was attenuated. Therefore, the activity of RNA polymerase, which is responsible for virus replication and transcription, was inhibited by the knockdown of CAMKII. This indicates that CAMKII may promote viral proliferation in the viral life cycle. Geniposide treatment at concentrations of 320 and $160 \mu \mathrm{g} / \mathrm{ml}$ remarkably decreased IAV polymerase activity; however, IAV polymerase activity was much more robust in CAMKII-deficient 293T cells (Fig. 4B). These results suggest that the inhibition of IAV replication by geniposide might be related to the calcium signaling pathway.

\subsection{Inhibitory effect of geniposide on influenza-induced $\mathrm{Ca}^{2+}$ influx}

Extracellular $\mathrm{Ca}^{2+}$ influx plays a pivotal role in IAV entry and subsequently mediates the activation of the calcium signaling pathway and facilitates IAV infection in the host cell. To explore the effect of geniposide on $\mathrm{Ca}^{2+}$ influx during the early stage of infection, MDCK cells were stained with fluo-3 AM dye, and the fluorescence intensity was determined by laser scanning confocal microscopy. In MDCK cells infected with IAV, $\left[\mathrm{Ca}^{2+}\right]$ increased significantly at $30 \mathrm{~min}$ after infection. Geniposide treatment at concentrations of 320,160 , and $80 \mu \mathrm{g} / \mathrm{ml}$ significantly inhibited the elevation in $\left[\mathrm{Ca}^{2+}\right]$ a a $30 \mathrm{~min}$ after infection, demonstrating that $\mathrm{Ca}^{2+}$ influx can be prevented by geniposide in the early stage of IAV replication (Figs. 5A and 5B).

\subsection{Effect of geniposide on the calcium signaling pathway in A549 cells infected by IAV}

PPI network construction and functional enrichment analysis showed that geniposide has a multi-target and multi-pathway inhibitory effect against influenza, and one of the mechanisms involves calcium. To verify the effect of geniposide on the calcium signaling pathway, the protein expressions of CAMKII, CREB, and c-Fos in A549 cells were determined at 24, 36, and $48 \mathrm{~h}$ after IAV infection.

CAMKII expression was markedly decreased at $12,24,36$, and $48 \mathrm{~h}$ after IAV infection. In contrast, the CREB and c-Fos expressions were significantly increased at 12, 24, 36, and $48 \mathrm{~h}$ after IAV infection. Geniposide treatment increased CAMKII expression in a dose-dependent manner at 12, 24, 36, and $48 \mathrm{~h}$ after IAV infection. Meanwhile, the CREB and c-Fos expressions were inhibited by geniposide at 12, 24,36 and $48 \mathrm{~h}$ after IAV infection (Figs. 6A-6D). The results demonstrate that the IAV-induced changes in the calcium signaling pathway were reversed by geniposide treatment.

\section{Discussion}


Current anti-influenza therapeutics and drugs under development directly target proteins encoded by the virus; for instance, oseltamivir inhibits neuraminidase, adamantanes block the M2 ion channel, and the nucleoside analog favipiravir targets RdRP [42]. Alternative antiviral approaches that target essential host proteins and pathways in the viral life cycle are appealing because they are effective against different virus strains and are less prone to resistance. Therefore, small-molecule inhibitors that target host factors may temporarily block the virus cycle without compromising cellular function.

Unique host genes have been confirmed to be critical for influenza virus replication. For example, host genes ADAMTS7, CPE, DPP3, MST1, and PRSS12 govern inflammation (NF-KB), cAMP/calcium signaling (CRE/CREB), and apoptosis [43]. Host factors BUB3, CCDC56, CLTC, CYC1, NIBP, ZC3H15, C14orf173, CTNNB1, and ANP32B specifically inhibit viral replication and transcription by decreasing the relative activity of viral RNA polymerase without affecting host protein synthesis [44]. A genome-wide CRISPR/Cas9 screen revealed that the host factors SLC35A1, C2CD4C, TRIM23, PIGN, CIC, JAK2, and PIAS3 are critical for the replication of intracellular pathogens [45].

The neuraminidase of the influenza virus is a viral surface glycoprotein that promotes the release of virions by cleaving sialic acid to facilitate viral release and spread in the respiratory tract [46]. To explore the inhibitory effect of geniposide on IAV, the neuraminidase activity in MDCK cells was analyzed at $48 \mathrm{~h}$ after infection. Geniposide significantly reduced the increase in neuraminidase activity after virus infection in a dose-dependent manner, providing further evidence that geniposide exerts anti-IAV activity.

Viral RNA-dependent RNA polymerase (vRNPs) consists of vRNA, the RNA polymerase complex (RdRp), and the nuclear protein, which are the smallest units of viral replication. Therefore, RNA polymerase is the key to viral replication [47]. CAMKII has been identified as an antiviral host factor that interacts with IAV polymerase. The interaction between CAMKII and IAV polymerase plays a pivotal role in the outcome of virus infection and antiviral immune response. In the current study, geniposide treatment greatly decreased the levels of RNA polymerase in HEK-293T cells infected with IAV. In a ribonucleoprotein (RNP) reconstitution assay, knocking down CAMKII in IAV-infected HEK-293T cells enhanced vRNA production. These results demonstrate that the inhibition of IAV replication by geniposide might be related to the calcium signaling pathway after virus infection.

PPI network analysis indicated that 17 genes associated with geniposide are also associated with influenza viruses: CTLA4, AKT1, IL6, JUN, RAF1, MAPK14, CASP3, PIK3CG, FOX01, NGF, MAPK8, BCL2, FASLG, KITLG, PTPN22, AQR, and gallocatechin gallate (GCG). Existing research indicates that the host genes of geniposide, including cytokines TNF-a, IFN-y, IL-6, IL-4, and IL-10, may be may related to the antiinfluenza activity of geniposide. Geniposide can reduce tissue damage by reducing the inflammatory cascade initiated by the cytokines TNF- $a$, INF- $y$, and IL-6 [35]. Moreover, geniposide can enhance the protective anti-inflammation mechanism and immune regulation by enhancing the expressions of IL-4 and IL-10, thereby having an anti-influenza effect $[48,49]$. Thus, the mechanism of geniposide's inhibition of viral propagation may be the downregulation of the expression of cleaved caspase 3 and the reduction of caspase3 activation. Thus, reduce exporting RNPs from the nucleus, which reduce the RNPs to be 
packaged into infectious progeny virions at the cell membrane. Purpose of inhibiting the influenza virus is achieved [50-52]. The following genes are related to influenza virus: CTLA4, AKT1, JUN, RAF1, PIK3CG, F0X01, BCL2, FASLG (FASL), MAPK8, MAPK14, PTPN22, GCG, NGF, and KITLG. According to the different functions of each gene, their mechanisms of inhibition on influenza virus can be divided into positive feedback and negative feedback.

IAV infection affects apoptosis in the early and late stages of infection. Among the positive feedback genes, Bcl-2 can act as an antagonist to cell death; in late infection, IAV infection pro-apoptotic by decreasing Bcl-2 [53]. Studies have shown that AKT3 induced by IAV can inhibit FoxO1, and the FoxO pathway can inhibit IAV infection by mediating anti-apoptosis and anti-inflammatory reactions [54]. MAPK8 and MAPK14 are important members of the mitogen-activated protein kinase (MAPK) family. MAPKs control a series of cell activities in innate immune response and participate in the regulation of cytokine gene expression and programmed cell death; thus, they are closely related to the prevention and treatment of influenza virus [55]. H1N1 infection induces early and significant NGF upregulation. The overexpression of NGF likely plays a neuroimmunomodulatory role in H1N1 infection. NGF acts on the nociceptive fibers innervating the lower respiratory tract, leading to enhanced neurogenic inflammation in infected lungs [56]. Differential genes such as KITLG, FOXP3, miR-451, IL-2, IL-10, IL-6, and TNF- $a$ are mainly involved in viral infection and the immune inflammatory response. These differential genes might play a role in preventing the host from being infected by viruses and exert immune regulatory effects in the cytoplasm [57]. Neuraminidase is one of two glycoproteins on the surface of influenza virus. Neuraminidase cleaves terminal sialic acid residues and promotes the release of virus from infected cells. Studies have shown that GCG has a strong inhibitory effect on neuraminidase, thereby affecting the spread of influenza virus [58].

Among the negative feedback genes, CTLA-4, PTPN22, and FASL are related to the immune system. CTLA-4, which is a receptor in T cells, plays a key role in the downregulation of antigen-activated immune response and takes charge of the steady state of the immune system through regulatory $T$ cells [59]. Thus, CTLA-4 plays a key role in the pathogenesis of influenza virus and the defense against virus infection [60]. Studies have shown that PTPN22 inhibits immune-induced T cell expansion/activation and the immune amplification of mouse T cells into peptides. PTPN22 encodes lymphoid phosphatase, which may diminish an individual's capacity to generate protective immunity against influenza virus. T cell activation contributes to protective immunity after influenza infection $[61,62]$. Therefore, high levels of this gene are not conducive to the prevention and treatment of influenza. The upregulation of Fas expression in virus-infected cells leads to enhanced apoptosis mediated by FasL. Fas-FasL is involved in the apoptosis of lymphocytes induced by human influenza virus H1N1-infected monocytes through direct contact between cells [63].

Other negative feedback genes that are not related to the immune system include AKT1, JUN, RAF1, and PIK3CG. IAV can activate Akt, trigger the intracellular PI3K/Akt signaling pathway, and promote cell entry, virus protein synthesis, and virus replication $[64,65]$. C-jun can be activated (phosphorylated) in the early stage of IAV infection [66]. The downregulation of C-jun significantly suppresses viral replication and 
mitigates the subsequent expression of inflammatory cytokines. Therefore, $\mathrm{C}$-jun plays an important role in virus infection and replication [51]. Based on the analysis of RNA synthesis in vitro, RAF-1 (RNA polymerase activator 1) and RAF-2 have been identified as host factors that can stimulate RNA synthesis in the influenza virus [67]. Therefore, reducing RAF-1 can inhibit the influenza virus from the early stage.

With the maturation of gene research, the above genes have been shown to have some anti-influenza functions. By increasing the levels of positive feedback genes and reducing the level of negative feedback genes, the influenza virus can be prevented and treated.

But a keyword search did not reveal evidence that AQR is related to the influenza virus by Pubmed.

The primary GO biological processes and KEGG pathways were identified by module enrichment analysis. The primary GO biological processes identified were related to cell communication (14.53\%), metabolism (13.41\%), transport (11.17\%), signal transduction (9.5\%), response external stimulus $(5.03 \%)$, and the viral life cycle $(0.84 \%)$; these may be the main mechanisms of the anti-influenza activity of geniposide. Nine identified GO biological processes were related to the functions of calcium ion and the associated signaling pathway: cellular calcium ion homeostasis; calcium ion homeostasis; cytosolic calcium ion homeostasis; the elevation of cytosolic calcium ion concentration; regulation of calcium ion transport; elevation of cytosolic calcium ion concentration during G-protein signaling; negative regulation of calcium ion transport via voltage-gated calcium channel activity; regulation of calcium ion transport via voltage-gated calcium channel activity; and negative regulation of calcium ion transport 3 . These processes have functions in cell homeostasis, cell communication, transport, ion transport, and signal transduction. The results indicate that calcium ions might play a pivotal role in the inhibition of influenza virus by geniposide.

CAMKII is a crucial protein in the calcium signaling network. Calcium signaling, which is activated by $\mathrm{Ca}^{2+}$, is a key regulator of IAV internalization and infection, and IAV has been shown to cause $\mathrm{Ca}^{2+}$ influx. Calcium signaling is a key regulator of IAV internalization which is a ubiquitously expressed calcium sensor that regulates diverse cellular functions. Functional enrichment analysis identified the calcium signaling pathway in Module 1; thus, geniposide may exert an anti-influenza effect by inhibiting calcium influx. Genome-wide RNAi screens confirmed that CAMKII is involved in the post-entry steps of influenza virus replication. A recent study highlighted that diltiazem, a calcium channel blocker, significantly inhibited viral production in human lung epithelial A549 cells (in vitro) and a reconstituted human airway epithelium model (ex vivo). Moreover, diltiazem treatment remarkably prevented mortality and reduced weight loss in mice infected with influenza $A(\mathrm{H} 1 \mathrm{~N} 1)$ pdm09; thus, diltiazem is a promising candidate for the treatment of influenza infections [68]. Diltiazem repurposing of drugs as Novel Influenza Inhibitors from Clinical Gene Expression Infection Signatures.

To clarify whether geniposide inhibits virus replication in a calcium-dependent manner, the activity of RNA polymerase, which is responsible for virus replication and transcription, was inhibited by the knockdown of CAMKII. The results indicated that CAMKII may promote viral proliferation during the viral life cycle. 
Geniposide treatment remarkably decreased IAV polymerase activity; however, IAV polymerase activity was remarkably more robust in CAMKII-defi2cient 293T cells. The results suggest that the inhibition of IAV replication by geniposide might be related to the calcium signaling pathway.

Extracellular $\mathrm{Ca}^{2+}$ influx plays a key role in the invasion of IAV by mediating the activation of the calcium signaling pathway and promoting IAV infection in host cells. To further investigate whether geniposide suppresses IAV replication by decreasing extracellular $\mathrm{Ca}^{2+}$ influx, MDCK cells were stained with fluo-3 AM dye, and the fluorescence intensity was determined by laser scanning confocal microscopy. Geniposide significantly inhibited the increase in [ $\left.\mathrm{Ca}^{2+}\right]$ in MDCK cells infected with IAV at 30 min after infection. Thus, geniposide exerted an antiviral effect by preventing $\mathrm{Ca}^{2+}$ influx at the early stage of the IAV replication cycle.

The CAMK cascade is important for many normal physiological processes. The misregulation of the CAMK cascade can lead to a variety of disease states of cell proliferation and apoptosis [69]. Numerous studies have linked the transcription factor CREB to the modulation of various inflammatory mediators. CREB signaling is an important cellular process that serves a variety of functions. Most notably, with respect to influenza infection, CREB signaling has been shown to activate protein kinase $A(P K A)$ and thus play a role in protein synthesis $[43,70]$. Because c-Fos is a CREB downstream expression factor, it also plays an important role in viral infection. Studies have shown that c-Fos expression is significantly increased after viral infection. Investigations of the underlying mechanisms demonstrated that c-Fos transcriptional activating protein can activate the IL- 6 and IL-8 promoters, so as to exert anti-IAV function[71, 72]. CAMKII induces the expression of the downstream factor CREB, and c-Fos plays a crucial role in $\mathrm{Ca}^{2+}$ influx after IAV infection. CAMKII activation leads to the inhibition of the downstream factor CREB and c-Fos activity, which leads to the inhibition of virus replication. This process is facilitated by the repression of IAV polymerase, which is an efficient way to suppress virus replication. The effect of geniposide on the $\mathrm{Ca}^{2+}$ signal transduction pathway may be related to the inhibition of the CAMKII downstream factor CREB and c-Fos activity.

To verify the inhibition of CaMKII and the activation of CREB and c-Fos induced by IAV infection, we evaluated the expressions of CAMKII, CREB, and c-Fos in infected A549 cells at 12, 24, 36, and $48 \mathrm{~h}$ after infection. Geniposide treatment markedly increased CAMKII expression and inhibited CREB and c-Fox expression at these four post-infection time points. These results provide evidence that geniposide exerts anti-IAV activity by ameliorating the changes to the calcium signaling pathway induced by IAV. These results also verify the related findings of the module enrichment analysis.

Collectively, the data from the current study are consistent with the results of the PPI network analysis. The findings indicate that geniposide suppresses IAV replication in vitro. These anti-IAV effects may be directly related to the inhibition of viral proliferation by host factors. Geniposide inhibits virus replication in a CAMKII-dependent manner, which prevents the over-activation of IAV polymerase induced by IAV infection. Taken together, our findings reveal a new facet of the mechanism by which geniposide inhibits IAV; geniposide inhibits IAV replication by disrupting the interplay between IAV RNA polymerase and 
CAMKII and regulating the changes in the calcium signaling pathway essential for IAV replication. The results of this study may pave the way for the development of new antiviral agents against influenza.

\section{Conclusion}

At present surveillance research has indicated that almost all circulating human IAVs are resistant to relative drugs. The outcomes of influenza pathogenesis depend on the interactions between the virus and the host cellular proteins along with the activation of signal transduction pathways. So the development of novel drugs targeting cellular components may be a promising strategy. Geniposide has diverse pharmacological activities. Accumulating evidence has demonstrated the antiviral properties of geniposide, which has been shown to inhibit infection by influenza A (H1N1) pdm09 virus and enterovirus 71 virus both in vitro and in vivo. And our previous study showed that the iridoid glycoside extracted from Gardeniae fructus inhibited extracellular Ca2+ influx induced by IAV; however, the precise mechanism of action remains unclear. There is no relevant report on "geniposide regulates calcium signaling pathway essential for Influenza A Virus Replication" at present. This study aimed to investigate the mechanism of geniposide on influenza A virus (IAV). Our conclusion shows that the potential targets and signaling pathways of geniposide in the IAV infection were predicted by network pharmacology analysis. According to the result of network pharmacology analysis, we validated the calcium signaling pathway induced by influenza A virus (IAV) and investigated the effect of geniposide extracted from Gardenia jasminoides Ellis on this pathway. These anti-IAV effects might be related to the disrupted interplay between IAV RNA polymerase and CAMKII and the regulation of the downstream calcium signaling pathway essential for IAV replication. Taken together, the findings reveal a new facet of the mechanism by which geniposide fights IAV in a way that depends on CAMKII replication. We finding the highlights of the new mechanism of the interactions between the virus and the host cellular proteins along with the activation of signal transduction pathways. The results of this study may pave the way for the development of new antiviral agents against influenza.

\section{Abbreviations}




\begin{tabular}{ll} 
IAV & influenza A virus \\
\hline GO & primary gene ontology \\
\hline KEGG & Kyoto Encyclopedia of Genes and Genomes \\
\hline vRNA & virusRNA \\
\hline PPI & protein-protein interaction \\
\hline MDCK & Madin-Darby canine kidney \\
\hline CAMKII & calcium/calmodulin-dependent protein kinase II \\
\hline CREB & cAMP response element-binding protein \\
\hline CRE/CREB & cAMP/calcium signaling \\
\hline vRNPs & Viral RNA-dependent RNA polymerase \\
\hline RdRp & RNA polymerase complex \\
\hline RNP & ribonucleoprotein \\
\hline GCG & gallocatechin gallate \\
\hline MAPK & mitogen-activated protein kinase \\
\hline RAF-1 & RNA polymerase activator 1 \\
\hline GO-BP & Gene ontology biological processes \\
\hline SEM & standard error of the mean \\
\hline
\end{tabular}

\section{Declarations}

\section{Ethics approval and consent to participate}

"Not applicable" in this section.

Consent for publication

"Not applicable" in this section.

\section{Availability of data and materials}

All data generated or analysed during this study are included in this published article [and its supplementary information files].

Conflict of Interest 
All authors declare that the research was conducted in the absence of any commercial or financial relationships that could be construed as a potential conflict of interest.

\section{Author Contributions}

SG and XC designed the study; LZ, LB, YW, MC, and ZG performed the antiviral experiments; YZ conducted the network pharmacology analysis; RZ, JS, YB, YS, and RY analyzed and interpreted the data; SG and LZ wrote the manuscript.

\section{Acknowledgements}

"Not applicable" in this section.

\section{Funding}

This project was funded by the following grants: National Natural Science Foundation of China (Nos. 81773977 and 81303290) and China Basic Research Projects of China Academy of Chinese Medical Sciences (No. ZZ0908034).

\section{References}

1. Tregoning, J.S., R.F. Russell, and E. Kinnear, Adjuvanted influenza vaccines. Hum Vaccin Immunother, 2018. 14(3): p. 550-564.

2. Palache, A., et al., Seasonal influenza vaccine dose distribution in 157 countries (2004-2011). Vaccine, 2014. 32(48): p. 6369-76.

3. Behzadi, M.A. and V.H. Leyva-Grado, Overview of Current Therapeutics and Novel Candidates Against Influenza, Respiratory Syncytial Virus, and Middle East Respiratory Syndrome Coronavirus Infections. Front Microbiol, 2019. 10: p. 1327.

4. Zhang, Y., et al., Targeting Hemagglutinin: Approaches for Broad Protection against the Influenza A Virus. Viruses, 2019. 11(5).

5. Jia, W., et al., A Novel Antigenic Drift of Avian Influenza A(H7N9) Virus in Poultry, China, 2018. J Infect Dis, 2019.

6. Jain, S., et al., Hospitalized patients with 2009 H1N1 influenza in the United States, April-June 2009. N Engl J Med, 2009. 361(20): p. 1935-44.

7. Smith, G.J., et al., Origins and evolutionary genomics of the 2009 swine-origin H1N1 influenza A epidemic. Nature, 2009. 459(7250): p. 1122-5.

8. Chiu, S.S., et al., Age-matched comparison of children hospitalized for 2009 pandemic H1N1 influenza with those hospitalized for seasonal H1N1 and H3N2. PLoS One, 2011. 6(7): p. e21837. 
9. Writing Committee of the, W.H.O.C.o.C.A.o.P.I., et al., Clinical aspects of pandemic 2009 influenza $A$ (H1N1) virus infection. N Engl J Med, 2010. 362(18): p. 1708-19.

10. Su, S., et al., Epidemiology, Evolution, and Pathogenesis of H7N9 Influenza Viruses in Five Epidemic Waves since 2013 in China. Trends Microbiol, 2017. 25(9): p. 713-728.

11. Zhu, H., et al., Emergence and development of H7N9 influenza viruses in China. Curr Opin Virol, 2016. 16: p. 106-113.

12. Generous, A., et al., Identification of putative interactions between swine and human influenza A virus nucleoprotein and human host proteins. Virol J, 2014. 11: p. 228.

13. Enkirch, T., et al., Identification and in vivo Efficacy Assessment of Approved Orally Bioavailable Human Host Protein-Targeting Drugs With Broad Anti-influenza A Activity. Front Immunol, 2019. 10: p. 1097.

14. Ison, M.G., Antiviral Treatments. Clin Chest Med, 2017. 38(1): p. 139-153.

15. Doll, M.K., et al., Safety and effectiveness of neuraminidase inhibitors for influenza treatment, prophylaxis, and outbreak control: a systematic review of systematic reviews and/or meta-analyses. J Antimicrob Chemother, 2017. 72(11): p. 2990-3007.

16. Deyde, V.M., et al., Surveillance of resistance to adamantanes among influenza $A(H 3 N 2)$ and A(H1N1) viruses isolated worldwide. J Infect Dis, 2007. 196(2): p. 249-57.

17. Gubareva, L.V., et al., Global update on the susceptibility of human influenza viruses to neuraminidase inhibitors, 2015-2016. Antiviral Res, 2017. 146: p. 12-20.

18. de Chassey, B., et al., Virus-host interactomics: new insights and opportunities for antiviral drug discovery. Genome Med, 2014. 6(11): p. 115.

19. Karlas, A., et al., Genome-wide RNAi screen identifies human host factors crucial for influenza virus replication. Nature, 2010. 463(7282): p. 818-22.

20. Ackerman, E.E., et al., Network-Guided Discovery of Influenza Virus Replication Host Factors. MBio, 2018. 9(6).

21. Dai, X., L. Zhang, and T. Hong, Host cellular signaling induced by influenza virus. Sci China Life Sci, 2011. 54(1): p. 68-74.

22. Zhao, M., L. Wang, and S. Li, Influenza A Virus-Host Protein Interactions Control Viral Pathogenesis. Int J Mol Sci, 2017. 18(8).

23. Fujioka, Y., et al., A Ca(2+)-dependent signalling circuit regulates influenza $A$ virus internalization and infection. Nat Commun, 2013. 4: p. 2763. 
24. Fujioka, Y., et al., A Sialylated Voltage-Dependent $\mathrm{Ca}(2+)$ Channel Binds Hemagglutinin and Mediates Influenza A Virus Entry into Mammalian Cells. Cell Host Microbe, 2018. 23(6): p. 809-818 e5.

25. Wang, Q., et al., [Anti-inflammatory mechanism of heat-clearing and detoxifying Chinese herbs]. Zhongguo Zhong Yao Za Zhi, 2018. 43(18): p. 3787-3794.

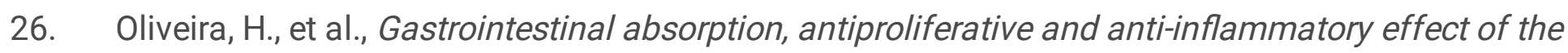
major carotenoids of Gardenia jasminoides Ellis on cancer cells. Food Funct, 2017. 8(4): p. 1672-1679.

27. Lee, E.J., J.K. Hong, and W.K. Whang, Simultaneous determination of bioactive marker compounds from Gardeniae fructus by high performance liquid chromatography. Arch Pharm Res, 2014. 37(8): p. 992-1000.

28. Li, C., et al., Geniposide protects against hypoxia/reperfusion-induced blood-brain barrier impairment by increasing tight junction protein expression and decreasing inflammation, oxidative stress, and apoptosis in an in vitro system. Eur J Pharmacol, 2019. 854: p. 224-231.

29. Rong, Y.P., et al., Protective Effects of Geniposide on Hepatic Ischemia/Reperfusion Injury. Transplant Proc, 2017. 49(6): p. 1455-1460.

30. Zhou, Y.X., et al., Diverse Pharmacological Activities and Potential Medicinal Benefits of Geniposide. Evid Based Complement Alternat Med, 2019. 2019: p. 4925682.

31. Li, N., et al., Antioxidative Property and Molecular Mechanisms Underlying Geniposide-Mediated Therapeutic Effects in Diabetes Mellitus and Cardiovascular Disease. Oxid Med Cell Longev, 2019. 2019: p. 7480512.

32. Zhang, X., T. Gao, and Y. Wang, Geniposide alleviates lipopolysaccharide (LPS)-induced inflammation by downregulation of miR-27a in rat pancreatic acinar cell AR42J. Biol Chem, 2019.

33. Zhang, Z., et al., Geniposide-mediated protection against amyloid deposition and behavioral impairment correlates with downregulation of mTOR signaling and enhanced autophagy in a mouse model of Alzheimer's disease. Aging (Albany NY), 2019. 11(2): p. 536-548.

34. Zhang, H.Y., et al., Antithrombotic activities of aqueous extract from Gardenia jasminoides and its main constituent. Pharm Biol, 2013. 51(2): p. 221-5.

35. Zhang, Y., et al., Geniposide demonstrates anti-inflammatory and antiviral activity against pandemic A/Jiangsu/1/2009 (H1N1) influenza virus infection in vitro and in vivo. Antivir Ther, 2017. 22(7): p. 599-611.

36. Lin, Y.J., et al., Inhibition of enterovirus 71 infections and viral IRES activity by Fructus gardeniae and geniposide. Eur J Med Chem, 2013. 62: p. 206-13. 
37. Son, M., et al., Genipin as a novel chemical activator of EBV lytic cycle. J Microbiol, 2015. 53(2): p. $155-65$.

38. Guo, S., et al., The inhibitory effect of iridoid glycoside extracted from Fructus Gardeniae on intracellular acidification and extracellular Ca2+ influx induced by influenza A virus. Exp Biol Med (Maywood), 2014. 239(8): p. 986-997.

39. Spackman, E. and M.L. Killian, Avian influenza virus isolation, propagation, and titration in embryonated chicken eggs. Methods Mol Biol, 2014. 1161: p. 125-40.

40. Spackman, E., et al., Assessment of replicate numbers for titrating avian influenza virus using dose-response models. J Vet Diagn Invest, 2019. 31(4): p. 616-619.

41. Wang, Z., et al., [Establishment of drug screening assay and pharmacodynamic evaluation method targeting influenza RNA polymerase]. Yao Xue Xue Bao, 2012. 47(9): p. 1159-63.

42. Goldhill, D.H., et al., The mechanism of resistance to favipiravir in influenza. Proc Natl Acad Sci U S A, 2018. 115(45): p. 11613-11618.

43. Meliopoulos, V.A., et al., MicroRNA regulation of human protease genes essential for influenza virus replication. PLoS One, 2012. 7(5): p. e37169.

44. Watanabe, T., et al., Influenza virus-host interactome screen as a platform for antiviral drug development. Cell Host Microbe, 2014. 16(6): p. 795-805.

45. Han, J., et al., Genome-wide CRISPR/Cas9 Screen Identifies Host Factors Essential for Influenza Virus Replication. Cell Rep, 2018. 23(2): p. 596-607.

46. Jin, Y.H., et al., Ethanolic Extract of Melia Fructus Has Anti-influenza A Virus Activity by Affecting Viral Entry and Viral RNA Polymerase. Front Microbiol, 2017. 8: p. 476.

47. $\mathrm{Li}, \mathrm{X}$., et al., U13 -> C13 mutation in the variable region of the NA gene 3'UTR of H9N2 influenza virus influences the replication and transcription of NA and enhances virus infectivity. Virus Genes, 2019. 55(4): p. 440-447.

48. Chen, J.Y., et al., Anti-inflammatory effects and pharmacokinetics study of geniposide on rats with adjuvant arthritis. Int Immunopharmacol, 2015. 24(1): p. 102-9.

49. Dai, M.M., et al., Effects and mechanisms of Geniposide on rats with adjuvant arthritis. Int Immunopharmacol, 2014. 20(1): p. 46-53.

50. Chen, Y., et al., Neuroprotective effects of geniposide in the MPTP mouse model of Parkinson's disease. Eur J Pharmacol, 2015. 768: p. 21-7.

51. J.Wurzer, W., et al., Caspase 3 activation is essential for efficient 
in`uenza virus propagation. 2003.

52. Jiang, Y.Q., et al., Geniposide Prevents Hypoxia/Reoxygenation-Induced Apoptosis in H9c2 Cells: Improvement of Mitochondrial Dysfunction and Activation of GLP-1R and the PI3K/AKT Signaling Pathway. Cell Physiol Biochem, 2016. 39(1): p. 407-21.

53. Mehrbod, P., et al., The roles of apoptosis, autophagy and unfolded protein response in arbovirus, influenza virus, and HIV infections. Virulence, 2019. 10(1): p. 376-413.

54. Wu, X., et al., A Hybrid Perturbative-Stochastic Galerkin Method for the Variability Analysis of Nonuniform Transmission Lines. IEEE Transactions on Electromagnetic Compatibility, 2020. 62(3): p. 746-754.

55. Dong, C., R.J. Davis, and R.A. Flavell, MAP kinases in the immune response. Annu Rev Immunol, 2002. 20: p. 55-72.

56. Chiaretti, A., et al., Interleukin and neurotrophin up-regulation correlates with severity of H1N1 infection in children: a case-control study. Int J Infect Dis, 2013. 17(12): p. e1186-93.

57. Lu, Q., et al., Molecular Characterization and Biological Function of a Novel LncRNA CRNG in Swine. Front Pharmacol, 2019. 10: p. 539.

58. Nguyen, T.T.H., et al., Inhibition effect of flavonoid compounds against neuraminidase expressed in Pichia pastoris. Biotechnol Bioprocess Eng, 2014. 19(1): p. 70-75.

59. Wang, X.B., et al., Regulation of surface and intracellular expression of CTLA-4 on human peripheral T cells. Scand J Immunol, 2001. 54(5): p. 453-8.

60. Ayukawa, H., et al., Expression of CTLA-4 (CD152) in peripheral blood T cells of children with influenza virus infection including encephalopathy in comparison with respiratory syncytial virus infection. Clin Exp Immunol, 2004. 137(1): p. 151-5.

61. Crabtree, J.N., et al., Autoimmune Variant PTPN22 C1858T Is Associated With Impaired Responses to Influenza Vaccination. J Infect Dis, 2016. 214(2): p. 248-57.

62. Hasegawa, K., et al., PEST domain-enriched tyrosine phosphatase (PEP) regulation of effector/memory T cells. Science, 2004. 303(5658): p. 685-9.

63. Nichols, J.E., J.A. Niles, and N.J. Roberts, Jr., Human lymphocyte apoptosis after exposure to influenza A virus. J Virol, 2001. 75(13): p. 5921-9.

64. Ehrhardt, C., et al., Influenza A virus NS1 protein activates the PI3K/Akt pathway to mediate antiapoptotic signaling responses. J Virol, 2007. 81(7): p. 3058-67. 
65. Shin, Y.K., et al., Influenza A virus NS1 protein activates the phosphatidylinositol 3-kinase (PI3K)/Akt pathway by direct interaction with the $p 85$ subunit of PI3K. J Gen Virol, 2007. 88(Pt 1): p. 1318.

66. Ludwig, S., et al., Influenza virus-induced AP-1-dependent gene expression requires activation of the JNK signaling pathway. J Biol Chem, 2001. 276(14): p. 10990-8.

67. Momose, F., et al., Cellular splicing factor RAF-2p48/NPI-5/BAT1/UAP56 interacts with the influenza virus nucleoprotein and enhances viral RNA synthesis. J Virol, 2001. 75(4): p. 1899-908.

68. Pizzorno, A., et al., Repurposing of Drugs as Novel Influenza Inhibitors From Clinical Gene Expression Infection Signatures. Front Immunol, 2019. 10: p. 60.

69. Colomer, J. and A.R. Means, Physiological roles of the Ca2+/CaM-dependent protein kinase cascade in health and disease. Subcell Biochem, 2007. 45: p. 169-214.

70. Andrews, C.S., et al., Nontypeable Haemophilus influenzae-Induced MyD88 Short Expression Is Regulated by Positive IKKK and CREB Pathways and Negative ERK1/2 Pathway. PLoS One, 2015. 10(12): p. e0144840.

71. Moreno, J.L., et al., Maternal influenza viral infection causes schizophrenia-like alterations of 5HT囚A and mG/u囚 receptors in the adult offspring. J Neurosci, 2011. 31(5): p. 1863-72.

72. Peng, N., et al., Inducible Major Vault Protein Plays a Pivotal Role in Double-Stranded RNA- or VirusInduced Proinflammatory Response. J Immunol, 2016. 196(6): p. 2753-66.

\section{Tables}

\section{Table 1 Modules from the PPI network}




\begin{tabular}{|c|c|c|c|c|}
\hline Module & Score & Node & Edge & Nodes Name \\
\hline 1 & 2.973 & 223 & 663 & $\begin{array}{l}\text { TAS2R60, CCR7, CCL4, CXCL8, CXCR5, CXCL10, CCR1, CCR3, } \\
\text { CXCL9, PF4, CCR2, CXCL1, CXCL12, CXCR3, CXCR4, CXCL11, } \\
\text { CXCL2, CCL4L1, CCL13, CCL20, CCL27, CXCL5, CX3CL1, PPBP, } \\
\text { CCL25, CCR4, CXCR2, CXCL3, CCL1, CXCR1, CXCL6, CX3CR1, } \\
\text { CCR10, CXCL16, CXCR6, C3AR1, CCR9, CCR8, CCL16, GNAI2, } \\
\text { APLN, ANXA1, APP, GPR183, AGT, AGTR2, ACKR3, S1PR1, } \\
\text { C5AR1, GNAI1, GNAI3, CCL28, FPR3, ADORA3, SAA1, FPR2, C3, } \\
\text { S1PR3, CASR, FPR1, S1PR2, GNAT3, OPRM1, PTGDR2, S1PR4, } \\
\text { KNG1, P2RY12, GNGT2, CNR1, S1PR5, SST, C5, OPRL1, POMC, } \\
\text { P2RY13, APLNR, CNR2, NPY, GNB3, CORT, NPY1R, NPY2R, DRD2, } \\
\text { DRD3, OPRK1, HCAR2, PTGER3, HRH4, GPR18, ADORA1, OPRD1, } \\
\text { GABBR1, GRM8, GNB4, GNG11, GNB2, GNB5, GNG4, GNG2, } \\
\text { TAS1R3,, GNB1, GRM2,, GNG7, P2RY14, PNOC, GNG10, GPR31, } \\
\text { GNG5, HCAR3, CHRM2, GNG12, GNGT1, GNG8, GNG13, GNG3, } \\
\text { PMCH, GRM6, GPSM3, GRM3, ADCY4, SUCNR1, LPAR5, LPAR2, } \\
\text { GRM4, GPR55, HCAR1, PENK, HTR1B, ADCY7, ADRA2A, LPAR3, } \\
\text { DRD4, OXER1, GRM7, GABBR2, PYY, LPAR1, BDKRB1, TAS2R7, } \\
\text { ADRA2C, GAL, ADRA2B, GPER11, PDYN, ADCY8, ADCY3, SSTR3, } \\
\text { HRH3, ADCY5, ADCY11, ADCY6, ADCY2, NMUR1, OXGR1, GPR11, } \\
\text { ADCY9, HTR1D, PSAP, NPBWR1, BDKRB2, MCHR1, MCHR2, } \\
\text { HTR1A, CHRM4, TAS2R39, TAS1 R1, TAS2R41, TAS2R38, GPR37, } \\
\text { TAS2R46, NPY5R, NPY4R, NPBWR2, NMUR2, P2RY4, PCP2, } \\
\text { SSTR5, SSTR2, SSTR4, HTR1F, MTNR1A, GALR2, TAS2R31, } \\
\text { TAS2R1, TAS1R2, TAS2R16, HTR5A, TAS2R14, HTR1E, GPSM2, } \\
\text { NMS, NMU, TAS2R42, TAS2R19, TAS2R10, TAS2R30, RXFP4, } \\
\text { TAS2R40, INSL5, MTNR1B, TAS2R50, RXFP3, TAS2R4, TAS2R3, } \\
\text { SSTR1, TAS2R5, GALR3, TAS2R20, TAS2R13, TAS2R9, NPB, } \\
\text { GPR37L1, PPY, HEBP1, TAS2R8, GALR1, GPSM1, TAS2R43, } \\
\text { CCR5, NPW, CCL5, RLN3, CCL19 }\end{array}$ \\
\hline 2 & 2.923 & 26 & 76 & $\begin{array}{l}\text { IKBKE, OASL, ISG15, IFIT1, OAS1, RSAD2, STAT1, TRIM25, IFIT3, } \\
\text { MX2, IFIT2, LY96, TRAF6, TRAF3, TBK1, IKBKG, PTPN11, UBBE2N, } \\
\text { TICAM2, TLR4, TANK, DDX58, CHUK, IRF7, UBE2V1, CD14 }\end{array}$ \\
\hline 3 & 1.5 & 6 & 9 & CFTR, PSMB9, NFKBIB, ITCH, NFKBIA, PSME3 \\
\hline 4 & 1.429 & 7 & 10 & LCK, B2M, PTAFR, FCGR1A, PIK3R2, PLCG1, HLA-DPA1 \\
\hline 5 & 1.4 & 5 & 7 & PYCARD, NLRC4, NLRP3, IL1B, CASP1 \\
\hline 6 & 1.2 & 5 & 6 & JAK3, IL7, IL2RG, IL2, MAPK1 \\
\hline 7 & 1 & 3 & 3 & IL17RC, IL17RA, IL17F \\
\hline 8 & 1 & 4 & 4 & CSF1, IL10, TIMP1, DNAJC3 \\
\hline 9 & 1 & 3 & 3 & NCR2, NCR1, NCR3 \\
\hline
\end{tabular}

Table 2 pathway of module 1 . 


\begin{tabular}{|c|c|c|}
\hline Pathway & Gene & $P$-value \\
\hline $\begin{array}{l}\text { Chemokine } \\
\text { signaling } \\
\text { pathway }\end{array}$ & $\begin{array}{l}\text { ADCY3, ADCY4, ADCY1, ADCY2, ADCY7, ADCY8, } \\
\text { ADCY5, ADCY6, CXCR1, CXCR2, CXCR3, CXCL11, } \\
\text { CXCL12, CXCL10, GNG8, CXCR5, CXCR4, CCR10, } \\
\text { CXCR6, GNG2, GNG3, GNG4, GNG5, GNG7, CCL4L1, } \\
\text { CCR9, CCR8, CCR7, PPBP, CCR5, GNB2, CCR4, GNB1, } \\
\text { CCR3, CCR2, CX3CR1, GNB5, GNB4, GNB3, CXCL1, } \\
\text { CCL1, GNAI3, CXCL5, GNAI2, GNAI1, CCR1, CXCL3, } \\
\text { CXCL2, CXCL9, GNG13, GNG11, PF4, CXCL6, CX3CL1, } \\
\text { GNG12, CCL5, CCL28, CCL4, CCL27, CCL25, CCL20, } \\
\text { CCL19, CCL16, GNGT1, GNGT2, CCL13, ADCY9, } \\
\text { CXCL16, GNG10 }\end{array}$ & $\begin{array}{l}1.657958766912765 \mathrm{E}- \\
55\end{array}$ \\
\hline $\begin{array}{l}\text { Neuroactive } \\
\text { ligand-receptor } \\
\text { interaction }\end{array}$ & $\begin{array}{l}\text { OPRM1, MCHR1, MCHR2, ADORA3, GABBR1, LPAR3, } \\
\text { LPAR2, GABBR2, LPAR1, ADORA1, S1PR2, S1PR3, } \\
\text { AGTR2, HTR1B, HTR1A, S1PR1, GALR1, NMUR1, } \\
\text { NMUR2, GALR3, S1PR4, GALR2, S1PR5, HTR1D, } \\
\text { HTR1F, HTR5A, HTR1E, PTGER3, C5AR1, NPBWR1, } \\
\text { NPBWR2, SSTR4, SSTR5, GRM4, GRM3, SSTR2, } \\
\text { GRM2, SSTR3, CHRM4, SSTR1, GRM8, CHRM2, } \\
\text { GRM7, GRM6, C3AR1, DRD3, DRD2, OPRK1, NPY2R, } \\
\text { DRD4, FPR1, FPR3, BDKRB1, FPR2, BDKRB2, APLNR, } \\
\text { HRH3, P2RY4, CNR1, CNR2, HRH4, ADRA2A, ADRA2C, } \\
\text { ADRA2B, OPRL1, NPY1R, NPY5R, P2RY13, P2RY14, } \\
\text { MTNR1B, MTNR1A, OPRD1 }\end{array}$ & $\begin{array}{l}9.657764704734148 \mathrm{E}- \\
49\end{array}$ \\
\hline $\begin{array}{l}\text { Taste } \\
\text { transduction }\end{array}$ & $\begin{array}{l}\text { ADCY4, TAS2R1, TAS2R4, ADCY8, TAS2R5, ADCY6, } \\
\text { TAS2R3, GNG13, TAS1R3, TAS1R1, TAS1R2, } \\
\text { TAS2R60, TAS2R46, TAS2R9, TAS2R42, TAS2R43, } \\
\text { TAS2R8, TAS2R7, GNG3, TAS2R20, TAS2R40, } \\
\text { TAS2R41, GNAT3, TAS2R16, GRM4, TAS2R13, } \\
\text { TAS2R39, TAS2R50, TAS2R14, TAS2R19, GNB1, } \\
\text { TAS2R38, TAS2R31, GNB3, TAS2R10 }\end{array}$ & $\begin{array}{l}2.0267715150108459 \mathrm{E}- \\
38\end{array}$ \\
\hline $\begin{array}{l}\text { Cytokine- } \\
\text { cytokine } \\
\text { receptor } \\
\text { interaction }\end{array}$ & $\begin{array}{l}\text { CXCL1, CCL1, CXCL5, CXCL3, CCR1, CXCL2, CXCL9, } \\
\text { CXCR1, PF4, CXCR2, CXCL6, CXCR3, CX3CL1, CCL5, } \\
\text { CXCL11, CCL4, CXCL 12, CCL28, CCL27, CXCL10, } \\
\text { CCL25, CXCR5, CCL20, CXCR4, CXCR6, CCR10, } \\
\text { CCL4L1, CCL19, CCL16, CCR9, CCR8, CCL13, CCR7, } \\
\text { PPBP, CCR5, CCR4, CCR3, CXCL16, CCR2, CX3CR1 }\end{array}$ & $\begin{array}{l}1.4758873776336994 \mathrm{E}- \\
15\end{array}$ \\
\hline Gap junction & $\begin{array}{l}\text { ADCY3, ADCY4, ADCY1, ADCY2, GNAI3, ADCY7, } \\
\text { GNAI2, DRD2, ADCY8, GNAI1, ADCY5, ADCY6, LPAR1, } \\
\text { ADCY9 }\end{array}$ & $9.270005860103292 \mathrm{E}-6$ \\
\hline Melanogenesis & $\begin{array}{l}\text { ADCY3, ADCY4, ADCY1, ADCY2, GNAI3, GNAI2, } \\
\text { ADCY7, ADCY8, GNAI1, ADCY5, ADCY6, POMC, } \\
\text { ADCY9 }\end{array}$ & $1.356035255661388 \mathrm{E}-4$ \\
\hline $\begin{array}{l}\text { Progesterone- } \\
\text { mediated } \\
\text { oocyte } \\
\text { maturation }\end{array}$ & $\begin{array}{l}\text { ADCY3, ADCY4, ADCY1, ADCY2, GNAI3, ADCY7, } \\
\text { GNAI2, ADCY9, GNAI1, ADCY8, ADCY5, ADCY6 }\end{array}$ & $\begin{array}{l}1.5948731269833238 \mathrm{E}- \\
4\end{array}$ \\
\hline $\begin{array}{l}\text { Intestinal } \\
\text { immune } \\
\text { network for IgA } \\
\text { production }\end{array}$ & CCR9, CCL25, CXCR4, CCR10, CCL28, CXCL12, CCL27 & 0.006412361067992899 \\
\hline
\end{tabular}




\begin{tabular}{|c|c|c|}
\hline $\begin{array}{l}\text { Dilated } \\
\text { cardiomyopathy }\end{array}$ & $\begin{array}{l}\text { ADCY3, ADCY4, ADCY1, ADCY2, ADCY7, ADCY9, } \\
\text { ADCY8, ADCY5, ADCY6 }\end{array}$ & 0.013743836429085802 \\
\hline $\begin{array}{l}\text { GnRH signaling } \\
\text { pathway }\end{array}$ & $\begin{array}{l}\text { ADCY3, ADCY4, ADCY1, ADCY2, ADCY7, ADCY9, } \\
\text { ADCY8, ADCY5, ADCY6 }\end{array}$ & 0.019520336471638235 \\
\hline $\begin{array}{l}\text { Complement } \\
\text { and coagulation } \\
\text { cascades }\end{array}$ & KNG1, C3AR1, C5AR1, C3, C5, BDKRB1, BDKRB2 & 0.031244059596336946 \\
\hline Oocyte meiosis & $\begin{array}{l}\text { ADCY3, ADCY4, ADCY1, ADCY2, ADCY7, ADCY9, } \\
\text { ADCY8, ADCY5, ADCY6 }\end{array}$ & 0.03592138311782549 \\
\hline $\begin{array}{l}\text { Vascular } \\
\text { smooth muscle } \\
\text { contraction }\end{array}$ & $\begin{array}{l}\text { ADCY3, ADCY4, ADCY1, ADCY2, ADCY7, ADCY9, } \\
\text { ADCY8, ADCY5, ADCY6 }\end{array}$ & 0.039354698598733834 \\
\hline $\begin{array}{l}\text { Calcium } \\
\text { signaling } \\
\text { pathway }\end{array}$ & $\begin{array}{l}\text { ADCY3, ADCY4, ADCY1, ADCY2, PTGER3, ADCY7, } \\
\text { ADCY9, ADCY8, CHRM2, BDKRB1, BDKRB2, HTR5A }\end{array}$ & 0.040643127061523385 \\
\hline
\end{tabular}

\section{Table 3 Pathway of module 2}

\begin{tabular}{|c|c|c|}
\hline Pathway & Gene & $P$-value \\
\hline $\begin{array}{l}\text { Toll-like receptor signaling } \\
\text { pathway }\end{array}$ & $\begin{array}{l}\text { IKBKE, TBK1, LY96, IRF7, IKBKG, TICAM2, } \\
\text { TLR4, TRAF6, STAT1, CHUK, CD14, } \\
\text { TRAF3 }\end{array}$ & $\begin{array}{l}1.2128632952757494 \mathrm{E}- \\
15\end{array}$ \\
\hline $\begin{array}{l}\text { RIG-I-like receptor signaling } \\
\text { pathway }\end{array}$ & $\begin{array}{l}\text { DDX58, IKBKE, ISG15, TBK1, IRF7, IKBKG, } \\
\text { TRIM25, TRAF6, CHUK, TANK, TRAF3 }\end{array}$ & $\begin{array}{l}2.6132796236858782 E- \\
15\end{array}$ \\
\hline $\begin{array}{l}\text { Cytosolic DNA-sensing } \\
\text { pathway }\end{array}$ & DDX58, IKBKE, TBK1, IRF7, IKBKG, CHUK & $6.896843891896822 \mathrm{E}-7$ \\
\hline Small-cell lung cancer & IKBKG, TRAF6, CHUK, TRAF3 & 0.0025018429161348032 \\
\hline $\begin{array}{l}\text { Pathogenic Escherichia coli } \\
\text { infection }\end{array}$ & LY96, TLR4, CD14 & 0.015072927052996406 \\
\hline $\begin{array}{l}\text { NOD-like receptor signaling } \\
\text { pathway }\end{array}$ & IKBKG, TRAF6, CHUK & 0.017685026048156277 \\
\hline $\begin{array}{l}\text { Adipocytokine signaling } \\
\text { pathway }\end{array}$ & IKBKG, CHUK, PTPN11 & 0.020476455840164474 \\
\hline Pathways in cancer & IKBKG, TRAF6, STAT1, CHUK, TRAF3 & 0.020691625131975113 \\
\hline $\begin{array}{l}\text { Epithelial cell signaling in } \\
\text { Helicobacter pylori } \\
\text { infection }\end{array}$ & IKBKG, CHUK, PTPN11 & 0.021055736236424673 \\
\hline Pancreatic cancer & IKBKG, STAT1, CHUK & 0.023441273355369472 \\
\hline Chronic myeloid leukemia & IKBKG, CHUK, PTPN11 & 0.02530096564417107 \\
\hline
\end{tabular}




\begin{tabular}{|lll|}
\hline Pathway & Gene & $P$-value \\
\hline Proteasome & PSME3, PSMB9 & 0.045385433295782915 \\
\hline
\end{tabular}

\section{Figures}

A

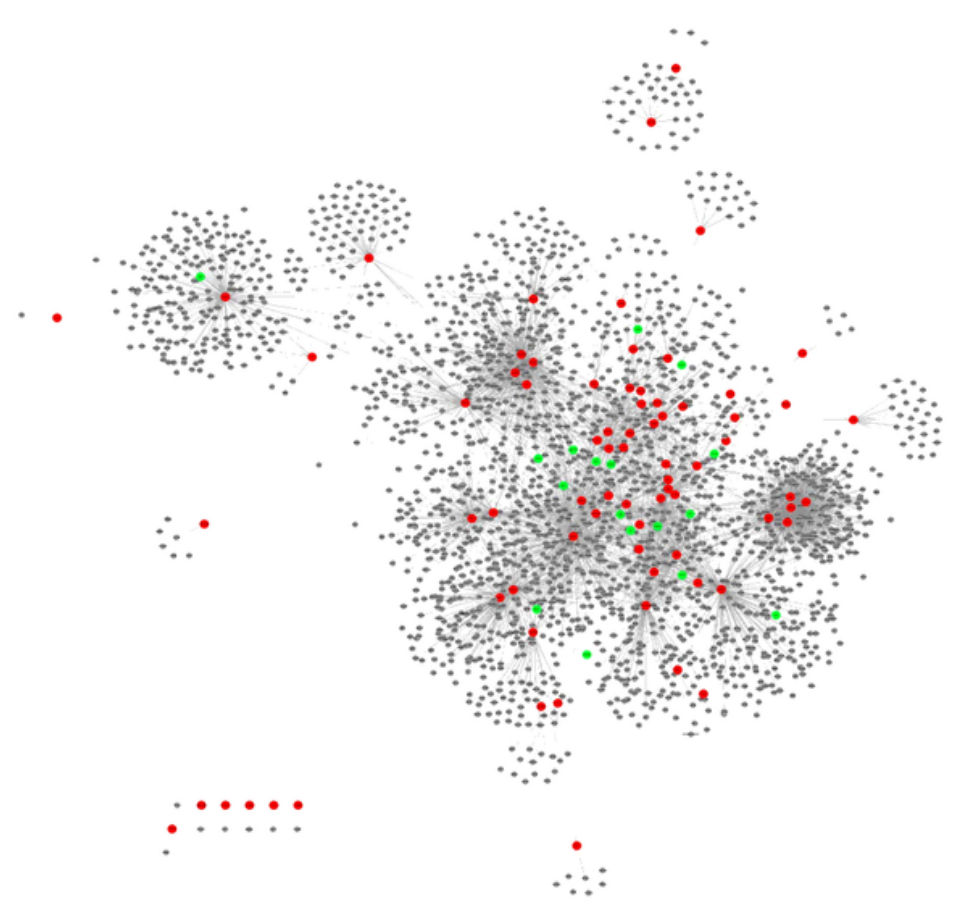

B
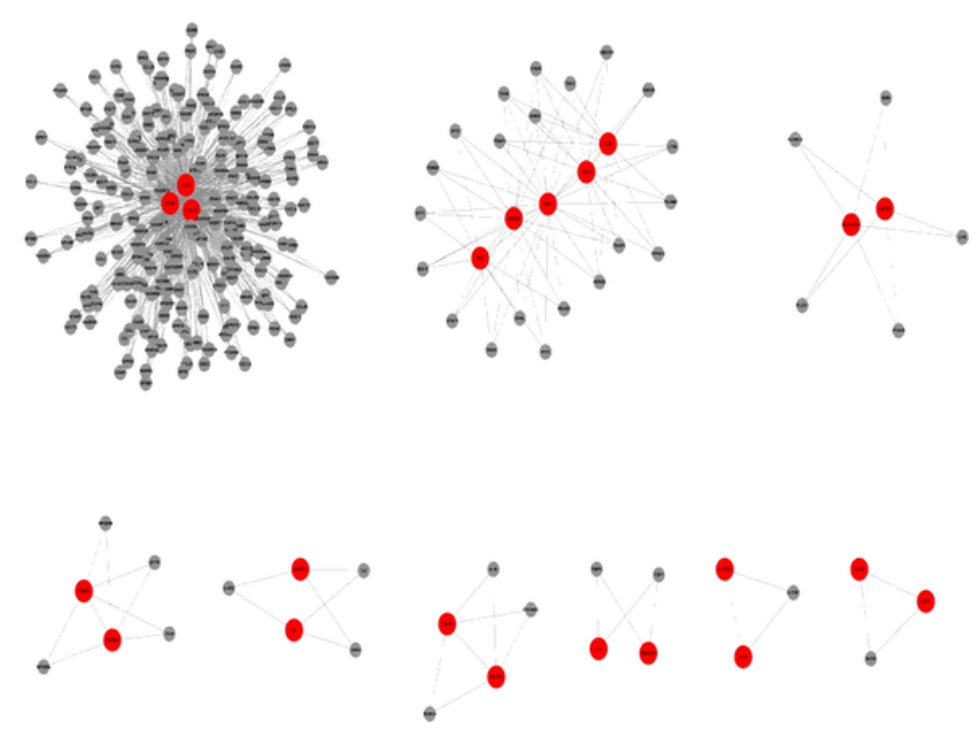

\section{Figure 1}

The network of " molecules-disease" of geniposide. Red nodes represent influenza virus-related molecules, gray nodes represent first-order neighbors of the influenza virus-related molecules, and green nodes represent geniposide-related molecules.

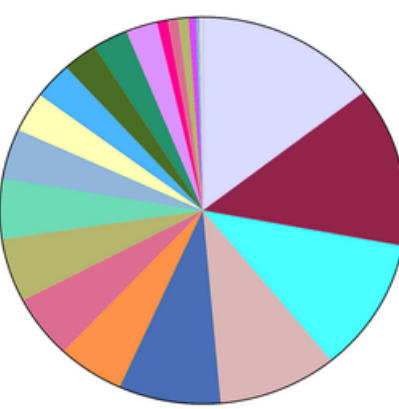

Module1

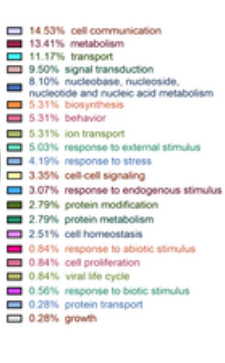

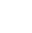

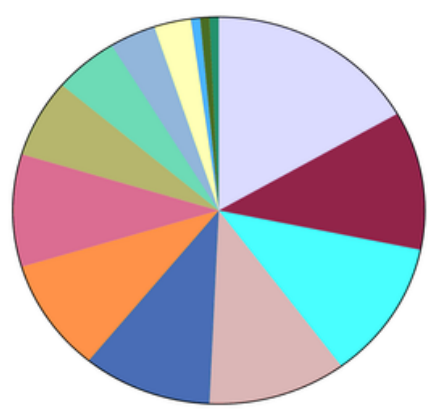

Module2

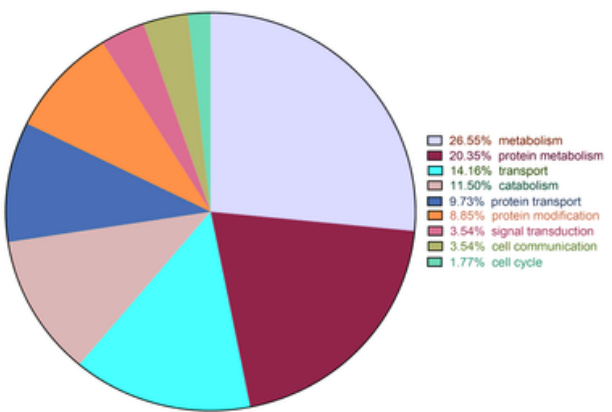

Module3

Figure 2

Gene ontology biological processes (GO-BP) classification chart of the three modules. 


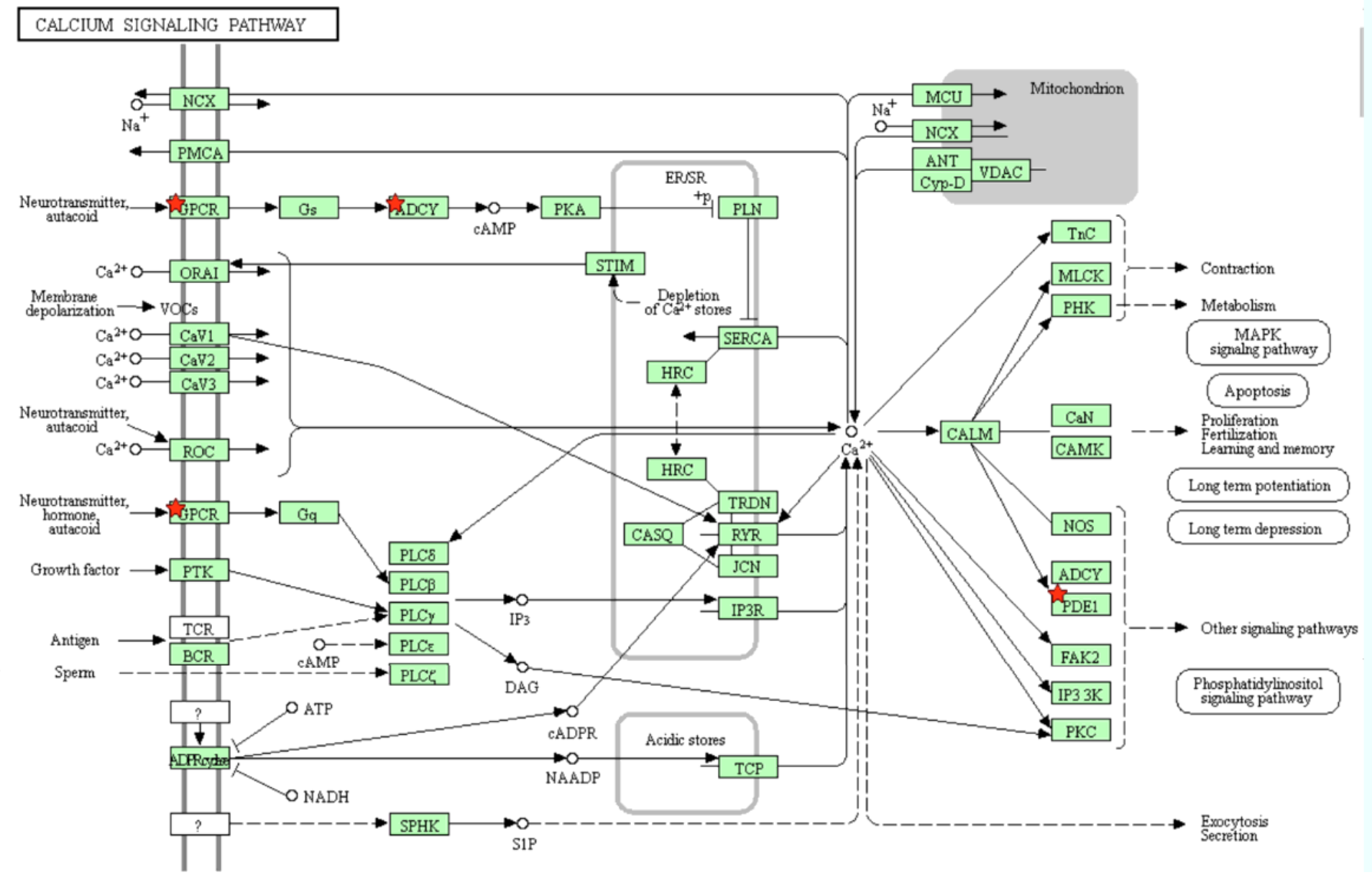

\section{Figure 3}

Overview of the calcium signaling pathway. The red stars are the molecules in the module that hit on the signaling pathway. 
A

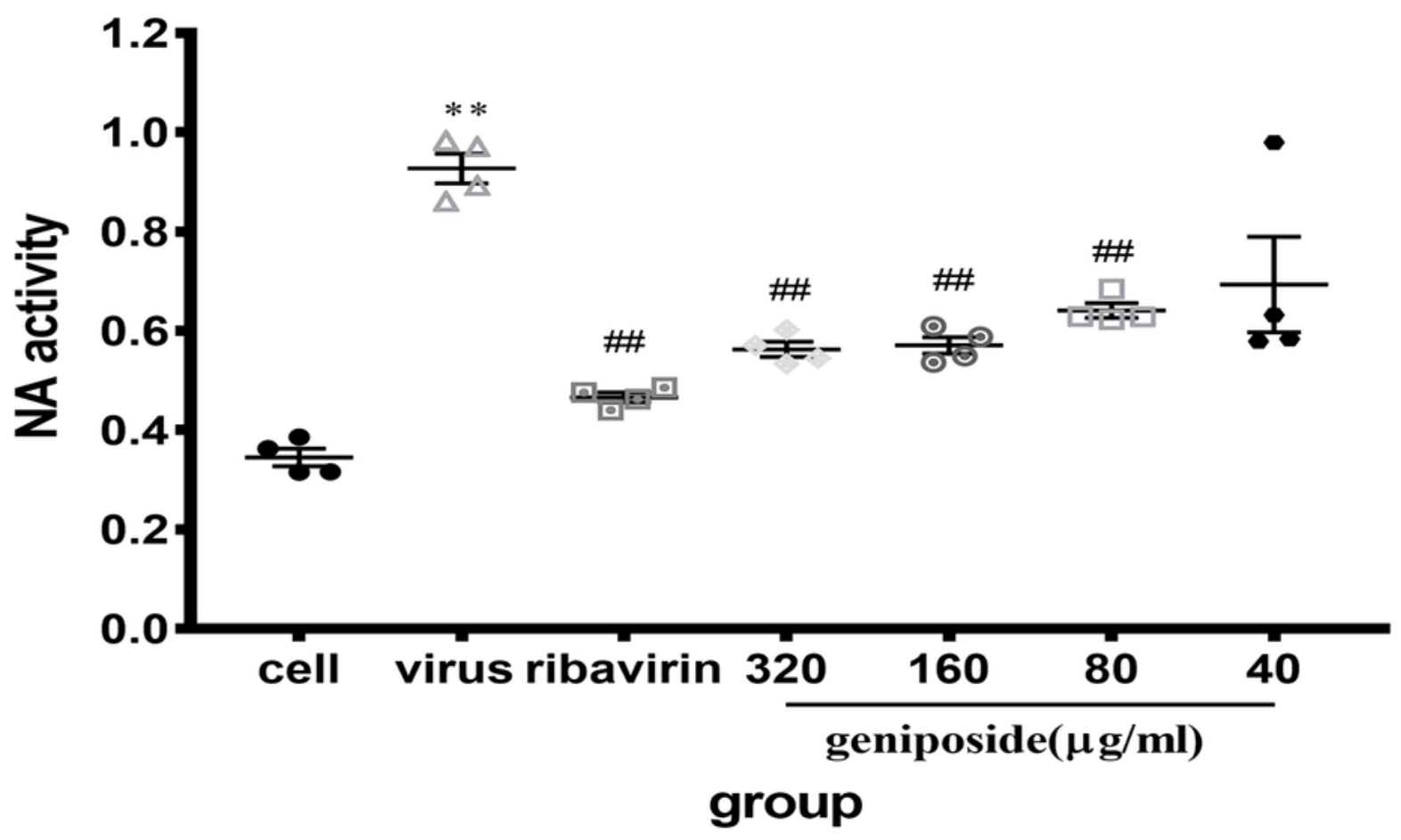

B

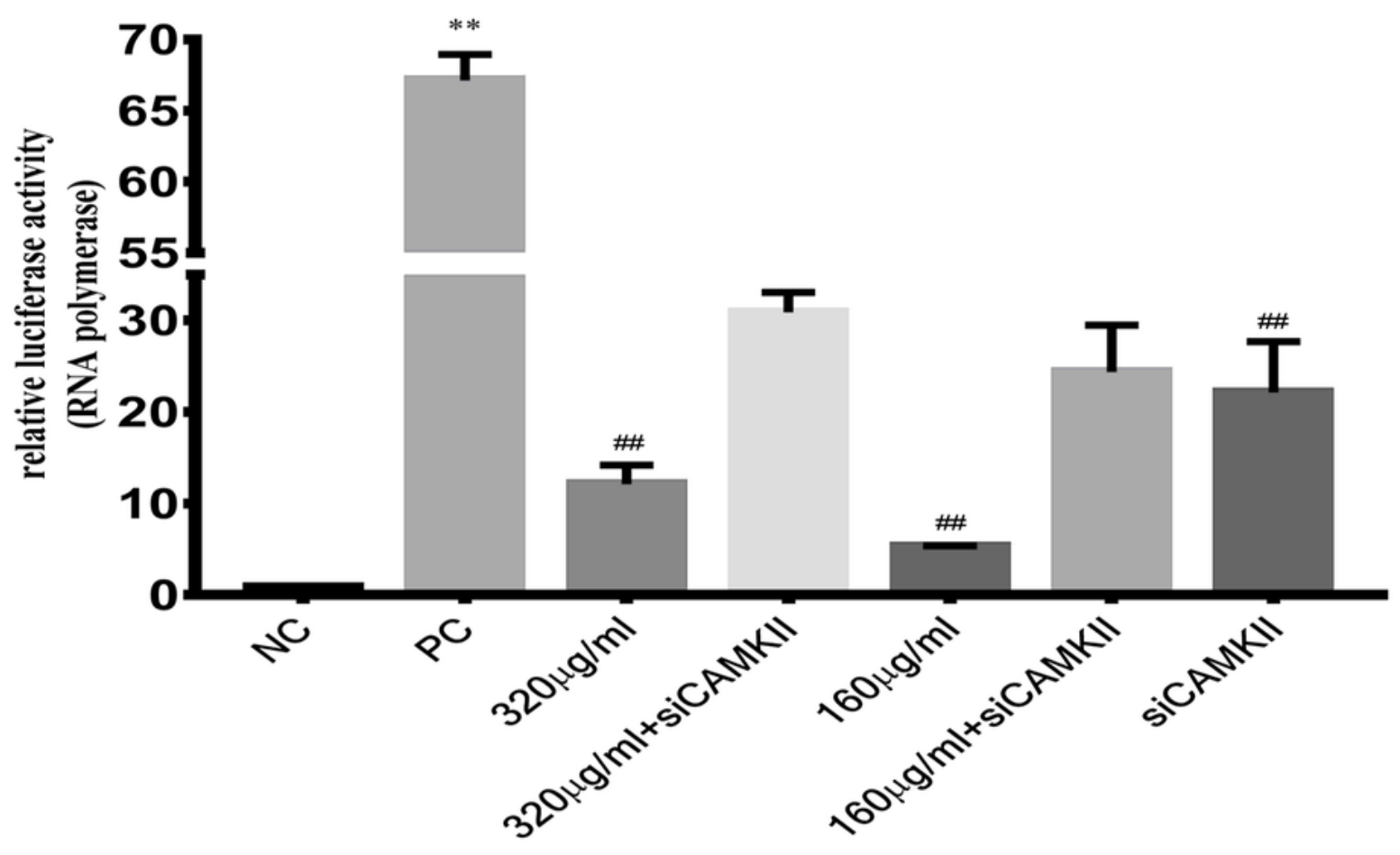

Figure 4

(A) Effect of geniposide on neuraminidase activity in MDCK cells infected with IAV. Values are expressed as mean \pm SEM $(n=4)$. ${ }^{*} P<0.01$ compared to the normal control group; \#\#P<0.01 compared to the virus control group. (B) Relative luciferase activity of IAV polymerase in HEK-293T cells. Relative luciferase activity was detected using a dual-luciferase reporter assay system. Values are expressed as 
mean \pm SEM $(n=4) .{ }^{*} P<0.01$ compared to the negative control group; \#\#P $<0.01$ compared to the positive control group.

A
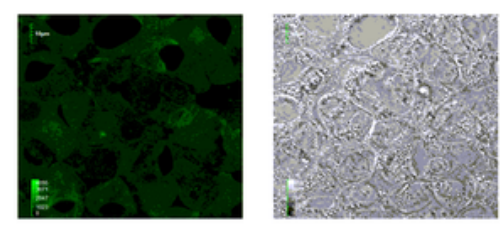

cell control
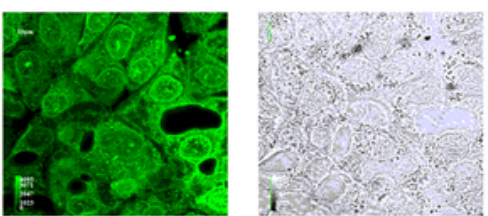

virus control
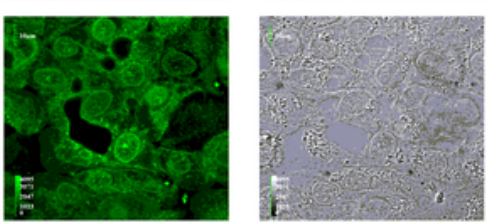

ribavirin control
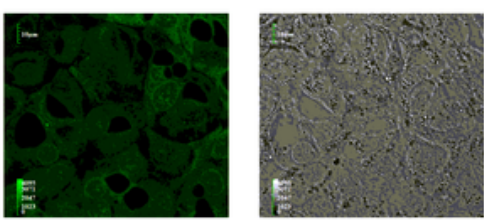

geniposide

$(320 \mu \mathrm{g} / \mathrm{ml})$
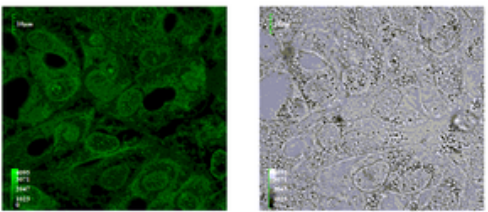

geniposide

$(160 \mu \mathrm{g} / \mathrm{ml})$
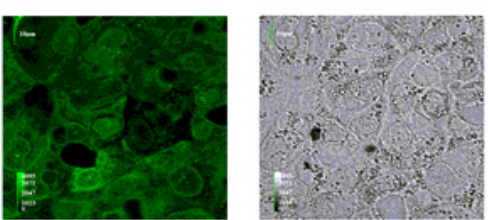

geniposide

$(80 \mu \mathrm{g} / \mathrm{ml})$

B

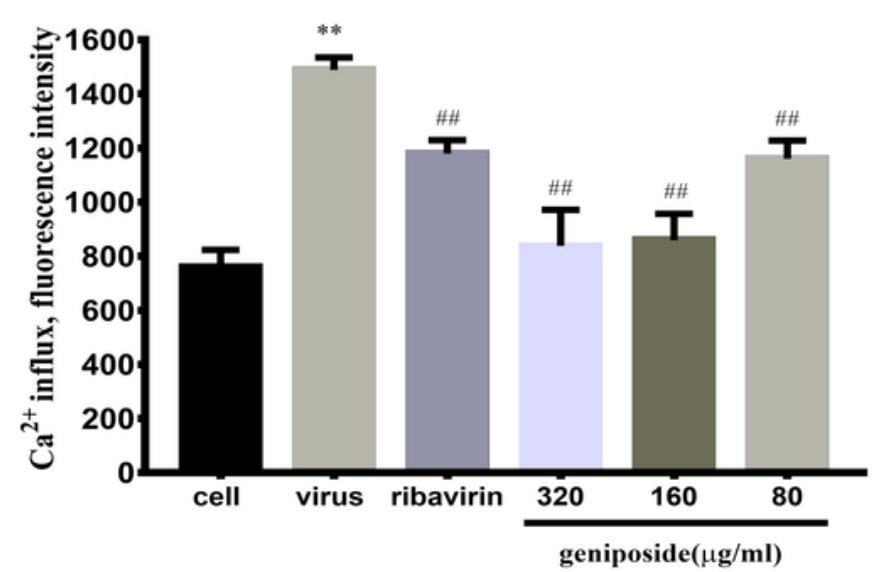

Figure 5

Effect of geniposide on [Ca2+]i levels in MDCK cells infected with IAV. Values are expressed as the mean fluorescence intensity of fluo-3 from four samples in each group. Eight randomly selected fields were analyzed in each sample. (A) Confocal images of MDCK cells stained with fluo-3 AM probe at $1 \mathrm{~h}$ after 
infection. Green images are fluorescence images taken at the emission wavelength of $530 \mathrm{~nm}$. Grayscale images are control images of MDCK cells without fluo-3 AM staining. (B) The concentration of [Ca2+]i is represented by the mean fluorescence intensity $(n=4)$. ${ }^{*} P<0.01$ compared to cell control group; \#\#P< 0.01 compared to infection control group. (A color version of this figure is available in the online journal.)

A
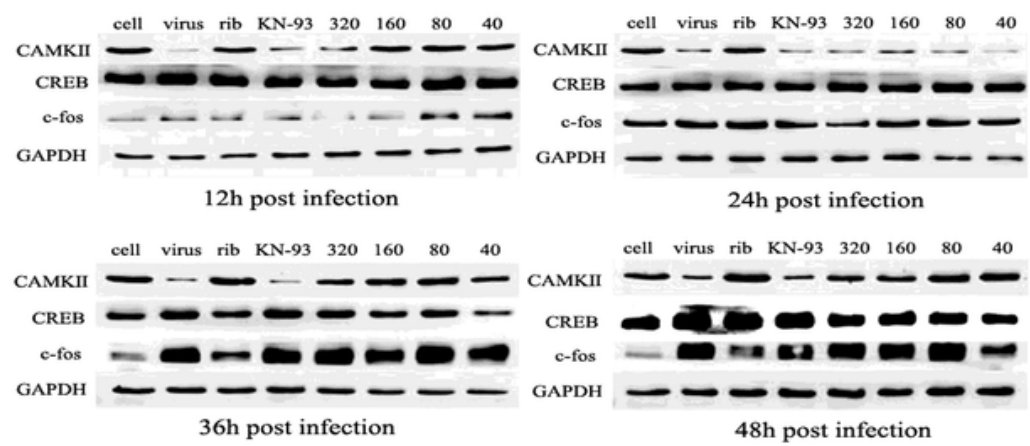

B

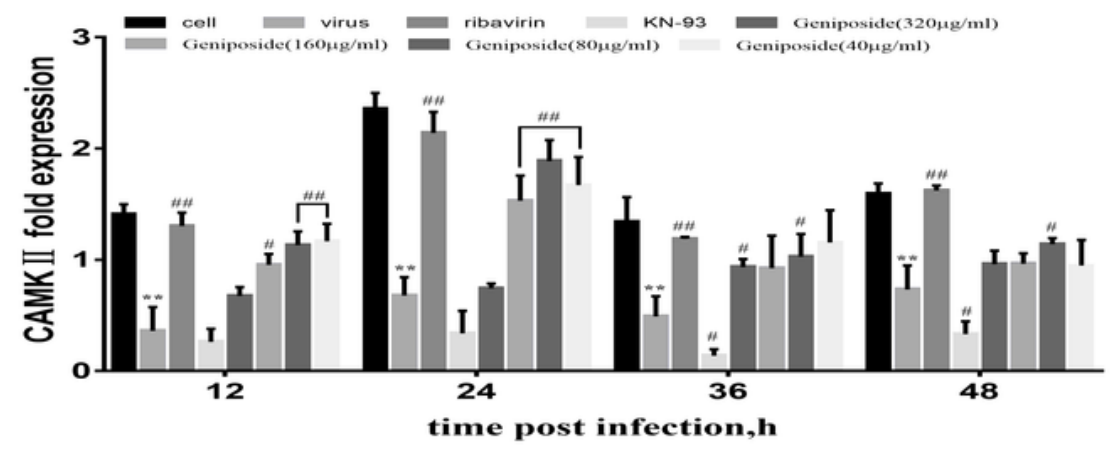

C

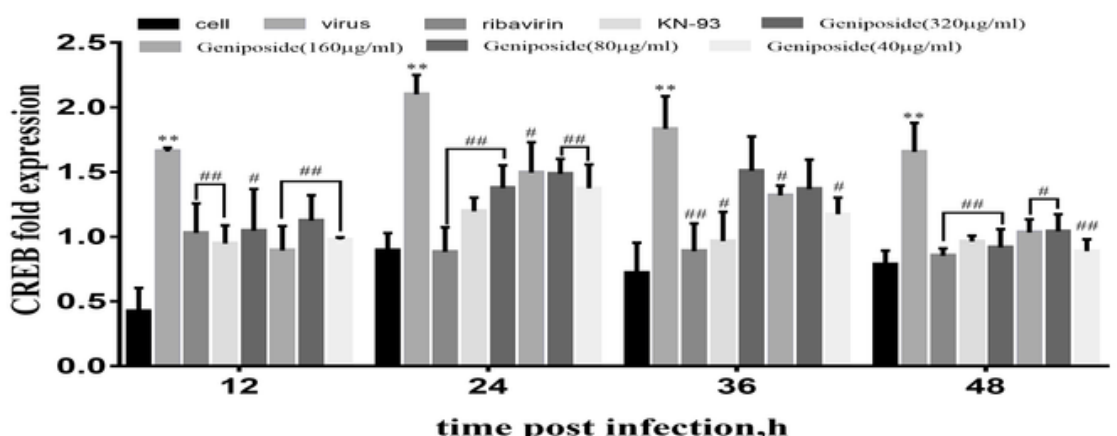

D

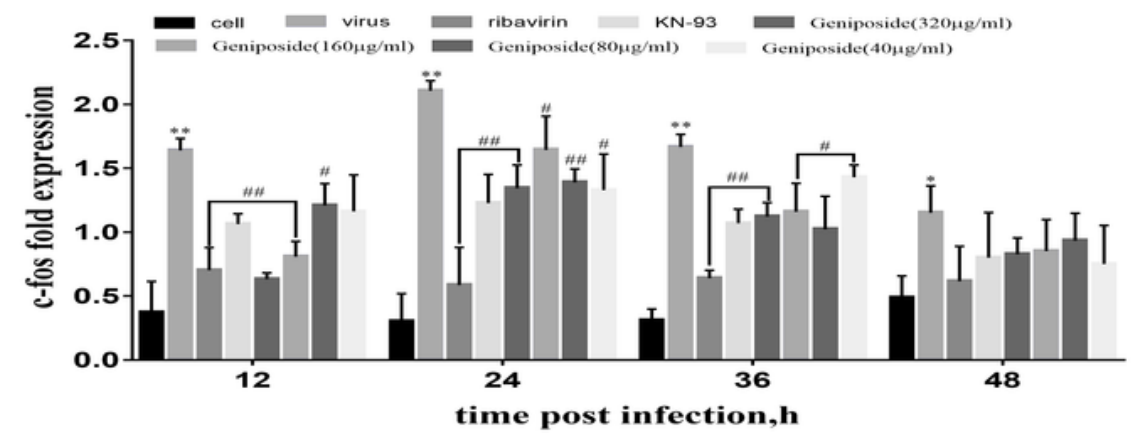

Figure 6 
Effects of geniposide on the expressions of CAMKII, CREB, and c-Fos activated by IAV in A549 cells. Values are expressed as the mean \pm SEM $(n=4) .{ }^{*} P<<0.01$ and ${ }^{*} P<0.05$ compared to the normal control group; \#\#P $<0.01$ and \#P $<0.05$ compared to the virus control group. (A) CAMKII, CREB, and c-Fos protein levels expressions were detected by western blotting in $A 549$ cells at 12, 24, 36, and $48 \mathrm{~h}$ after infection. (B-D) WB quantification of CAMKII, CREB, and c-Fos. The relative expressions of CAMKII, CREB, and c-Fos were calculated as the ratios of CAMKII, CREB, and c-Fos to GAPDH. 